PUPT-1279

IASSNS-HEP-91/60

September 1991

\title{
Scattering at Planckian Energies
}

\author{
HERMAN VERLINDE⿵冂卄 \\ Joseph Henry Laboratories \\ Princeton University \\ Princeton, NJ 08544 \\ and \\ ERIK VERLINDE[ \\ School of Natural Sciences \\ Institute for Advanced Study \\ Princeton, NJ 08540
}

\begin{abstract}
We give a systematic analysis of forward scattering in 3+1-dimensional quantum gravity, at center of mass energies comparable or larger than the Planck energy. We show that quantum gravitational effects in this kinematical regime are described by means of a topological field theory. We find that the scattering amplitudes display a universal behaviour similar to two dimensional string amplitudes, thereby recovering results obtained previously by 't Hooft. Finally, we discuss the two-particle process in some detail.
\end{abstract}

*Supported by NSF Grant PHY90-21984.

†'Supported by the W.M. Keck Foundation. 


\section{Introduction}

Quantum gravitational effects grow large when the length and time scales of the physical process of interest fall below the Planck length $\ell_{p l}$. This well-known fact has the following, necessarily heuristic, explanation. If we decide to keep our coordinates dimensionless, the metric $g_{\mu \nu}$ has dimension (length) ${ }^{2}$. This dimensionality can be made explicit by introducing some scale $L$, which we may take to be some length characteristic of the physical situation we wish to consider, and write

$$
g_{\mu \nu}=L^{2} \hat{g}_{\mu \nu}
$$

where $\hat{g}_{\mu \nu}$ is dimensionless. The Einstein action

$$
S_{E}[g]=\int \sqrt{g} R
$$

has the dimension of (length) ${ }^{2}$, that is

$$
S_{E}\left[L^{2} \hat{g}\right]=L^{2} S_{E}[\hat{g}]
$$

Now, although in the quantum theory we are supposed to integrate over all metrics, it seems physically reasonable to suppose that in this integral only those metrics are relevant whose size is comparable to that of the physical system. In other words, we may reasonably assume that the rescaled metric $\hat{g}_{\mu \nu}$ is typically of order 1 . With this assumption, we can now indeed conclude from (1.3) that, since $S_{E}$ is normalized

by Newton's constant $G=\ell_{p l}^{2}$, the dimensionless coupling constant determining the strength of the quantum gravitational effects is given by the ratio

$$
g(L)=\frac{\ell_{p l}}{L}
$$

of the Planck length $\ell_{p l}$ and the characteristic length $L$.

Because of this reasoning, it is generally believed that to predict scattering processes in which the center of mass energy exceeds the Planck energy, the full theory of quantum gravity (or perhaps string theory) has to be invoked. However, this is not necessarily true, since a scattering process between two particles is characterized 
by two energies, i.e. the center of mass energy and the momentum transfer, and even if the former is very large, the latter can be small. Therefore, in the physical context of very high energy collisions, we can regard quantum gravity as a theory with two coupling constants.

This paper is an attempt to exploit this fact to start a systematic study of high energy scattering in quantum gravity, in the limit where the momentum transfer is small compared to the Planck energy. This study was motivated by recent work by 't Hooft, who argued that in this kinematical regime quantum gravity can be treated semi-classically, and furthermore displays a remarkable formal resemblance to string theory [1, 2]. Both results will be confirmed by our analysis. We will explain why at small momentum transfer indeed all physical modes of the gravitational field become classical, except for two longitudinal modes. We then show that the quantum dynamics of these modes is described by means a topological field theory, which can be characterized as a two-dimensional topological sigma model with the group of diffeomorphisms in $1+1$ dimensions as target space. We will further study the interaction of this model with ultra-energetic particles and show that the amplitudes describing the forward scattering of these particles can be rewritten in a form similar to $1+1$ dimensional string amplitudes. We will end with a discussion of the twoparticle process.

\section{The Einstein Action with Two Coupling Constants}

To characterize the scattering processes that we are interested in, let us imagine that some day the technology will be available to build a linear accelerator that can accelerate elementary particles, say electrons, to energies comparable or larger than the Planck energy. For concreteness, let us choose our $x$ axis along this accelerator, and introduce the notation $x^{\alpha} \equiv(t, x)$ for the longitudinal and $y^{i} \equiv(y, z)$ for the transversal coordinates. Thus the momenta of the particles are of Planckian magnitude in the $x^{\alpha}$-plane, while the perpendicular momenta $p_{i}$ are negligible. Now, since the transversal cross sections of the electron beams used in our Gedanken experiments are necessarily very large compared to the Planck length, in practice all collisions between the left- and right-moving electrons have relatively very large impact parameters. Hence we are dealing almost exclusively with forward scattering. 
The physics of these forward scattering processes is characterized by two length scales of very different order of magnitude, namely $\ell_{/ /}$, the typical longitudinal wavelength of the particles, and $\ell_{\perp}$, the characteristic length of the impact parameter. Hence, we can form the two dimensionless ratios

$$
\begin{aligned}
& g_{/ /} \equiv \frac{\ell_{p l}}{\ell_{/ /}} \sim 1 \\
& g_{\perp} \equiv \frac{\ell_{p l}}{\ell_{\perp}} \ll 1 .
\end{aligned}
$$

We will now show that, in this regime, quantum gravity indeed naturally splits into two sectors, one strongly coupled with $g_{/ /}$, and one weakly coupled with $g_{\perp}$.

\subsection{Splitting the Einstein Action}

Given the above geometrical setup, it is natural to make the following gauge choice

$$
g_{\mu \nu}=\left(\begin{array}{cc}
g_{\alpha \beta} & 0 \\
0 & h_{i j}
\end{array}\right) .
$$

Here $g_{\alpha \beta}$ has the Lorentzian signature, and $h_{i j}$ is Euclidean. It is evident that locally the metric can always be brought in this form. This gauge may look somewhat unfamiliar, and indeed, it is easy to see that it is not suitable for doing perturbative calculations around an (asymptotically) flat background. Our interest, however, is in doing non-perturbative calculations in $g_{/ /}$, and for this purpose it is sufficient to know that the above gauge choice is non-singular around sufficiently generic metrics. Further, in general there may be global obstructions to choosing the metric of the above form, but these do not occur in our situation, because we assume that the topology in the $x^{\alpha}$ and $y^{i}$ direction is trivial.

The physical motivation for choosing the gauge (2.2) is that in the kinematical regime of interest, the characteristic scale $\ell_{/ /}$of $g_{\alpha \beta}$ is of the order of $\ell_{p l}$, while the typical length $\ell_{\perp}$ of $h_{i j}$ is much larger. Thus, following the line of argument given in the Introduction, let us introduce two dimensionless metrics $\hat{g}_{\alpha \beta}$ and $\hat{h}_{i j}$ via

$$
\begin{aligned}
g_{\alpha \beta} & =\ell_{/ /}^{2} \hat{g}_{\alpha \beta}, \\
h_{i j} & =\ell_{\perp}^{2} \hat{h}_{i j} .
\end{aligned}
$$

We again will make the plausible assumption that $\hat{g}$ and $\hat{h}$ are of order 1 . 
Now, a straightforward calculation, outlined in the Appendix, shows that the Einstein action in the gauge (2.2) splits into two terms

$$
S_{E}[g]=S_{/ /}[g, h]+S_{\perp}[h, g]
$$

given by

$$
S_{/ /}[g, h]=\int \sqrt{g}\left(\sqrt{h} R_{h}+\frac{1}{4} \sqrt{h} h^{i j} \partial_{i} g_{\alpha \beta} \partial_{j} g_{\gamma \delta} \epsilon^{\alpha \gamma} \epsilon^{\beta \delta}\right)
$$

and

$$
S_{\perp}[h, g]=\int \sqrt{h}\left(\sqrt{g} R_{g}+\frac{1}{4} \sqrt{g} g^{\alpha \beta} \partial_{\alpha} h_{i j} \partial_{\beta} h_{k l} \epsilon^{i k} \epsilon^{j l}\right)
$$

The crucial property separating both terms is that they behave differently under the constant rescaling (2.3) of $g_{\alpha \beta}$ and $h_{i j}$, namely

$$
\begin{aligned}
& S_{/ /}\left[\ell_{/ /}^{2} \hat{g}, \ell_{\perp}^{2} \hat{h}\right]=\ell_{/ /}^{2} S_{/ /}[\hat{g}, \hat{h}], \\
& S_{\perp}\left[\ell_{\perp}^{2} \hat{h}, \ell_{/ /}^{2} \hat{g}\right]=\ell_{\perp}^{2} S_{\perp}[\hat{h}, \hat{g}] .
\end{aligned}
$$

Thus, after we make $S_{E}$ dimensionless by dividing by Newton's constant $G=\ell_{p l}^{2}$, we see that the $S_{/ /}$part of the Einstein action is strongly coupled, with coupling $g_{/ /}=\ell_{p l} / \ell_{/ /}$, whereas the coupling constant $g_{\perp}=\ell_{p l} / \ell_{\perp}$ of the other term $S_{\perp}$ is very small. We arrive therefore at the important conclusion that as far as $S_{\perp}$ is concerned we are in the classical regime. In the following we will exploit this simplification of the dynamics of quantum gravity to analyze the theory to leading order in $g_{\perp}$, while keeping track of the full non-perturbative dependence on $g_{/ /}$.

To complete the picture we should include the ghost action associated with our gauge choice $g_{i \alpha}=0$. Under an infinitesimal coordinate transformation we have $\delta g^{i \alpha}=h^{i j} \partial_{j} \xi^{\alpha}+g^{\alpha \beta} \partial_{\beta} \xi^{i}$, so we find that the ghost action consists of the two terms

$$
\begin{aligned}
S_{g h}[b, c] & =S_{/ /}[b, c]+S_{\perp}[b, c] \\
S_{/ /}[b, c] & =\int \sqrt{g} \sqrt{h} h^{i j} b_{i \alpha} \partial_{j} c^{\alpha}, \\
S_{\perp}[b, c] & =\int \sqrt{h} \sqrt{g} g^{\alpha \beta} b_{i \alpha} \partial_{\beta} c^{i} .
\end{aligned}
$$

Again we observe that after the rescaling (2.3) the first term $S_{/ /}$is strongly coupled and the second $S_{\perp}$ weakly.

*The $\epsilon$-symbols in (2.5) and (2.6) contain a factor of $(\sqrt{g})^{-1}$ resp. $(\sqrt{h})^{-1}$, so that they transform as tensors. 


\subsection{Isolating the Relevant Gravitational Modes}

It is instructive to make an inventory of the different degrees of freedom of the gravitational field in relation with the above decomposition of the action. As usual in quantum gravity we have, after gauge-fixing, 6 remaining components of the metric and 4 anti-commuting ghost variables. The ghosts eliminate the 4 longitudinal modes of the metric via the BRST-invariance of the action and effectively impose the 'Gauss law' constraints

$$
R_{i \alpha}=0
$$

This leaves 2 physical degrees of freedom constituting the gravitational radiation modes. A useful parametrization of the metric variables, which manifestly exhibits the different modes, is

$$
\begin{aligned}
g_{\alpha \beta} & =e^{\phi(X, Y)} \partial_{\alpha} X^{a} \partial_{\beta} X^{b} \eta_{a b} \\
h_{i j} & =e^{\chi(X, Y)} \partial_{i} Y^{p} \partial_{j} Y^{q} \delta_{p q} .
\end{aligned}
$$

Here $X^{a}(x, y)$ and $Y^{p}(x, y)$ are the diffeomorphisms that relate $g_{\alpha \beta}$ resp. $h_{i j}$ to the diagonal metrics. As such, they are paired with the ghost fields $c^{\alpha}$ resp. $c^{i}$ via the BRST symmetry. Hence they are the longitudinal modes and locally decouple from physical processes; as we will see, however, their global variations are important observables. The conformal factors $\phi$ and $\chi$ are physical, BRST-invariant fields and represent the two transversal components of the graviton.

To see which of these modes are physically relevant in our context, let us consider the coupling to matter. In general, we can include matter by adding a source term to the Einstein action

$$
S_{E}[g] \rightarrow S_{E}[g]+S_{m}[g] .
$$

This source term represents the stress-energy of the matter particles, of which the transversal components $T_{i j}$ are assumed to be negligible compared to longitudinal components

$$
T_{\alpha \beta}=\frac{1}{\sqrt{g}} \frac{\delta S_{m}[g]}{\delta g^{\alpha \beta}} .
$$

Here $T_{\alpha \beta}$ is effectively traceless, provided the rest-masses of the matter particles are very small compared to the Planck mass. The remaining two components of $T_{\alpha \beta}$ can be identified with the momenta of the particles, which are either left- or right-moving. We see therefore that the matter stress energy only couples to the off-diagonal variations of $g_{\alpha \beta}$, which are parametrized by the fields $X^{a}$. 
An important property of $S_{\perp}$ is that it is invariant under reparametrizations $x^{\alpha} \rightarrow$ $\tilde{x}^{\tilde{\alpha}}(x, y)$. As a consequence, one finds that upon inserting the parametrization (2.10) into (2.5) and (2.6) that $S_{\perp}$ does not depend on $X^{a}$. Now, since $g_{\perp} \ll 1$, the functional integral over metrics is strongly peaked near the space of field configurations that minimize $S_{\perp}$, and thus to leading order in $g_{\perp}$, we can restrict the configuration space of gravity to this vacuum subspace. Combined with the above observation that the matter only couples to the $X^{a}$ fields, this shows that all other variables can be treated classically. The $X^{a}$ are therefore the relevant quantum mechanical modes, since their dynamics is dictated purely by the strongly coupled $S_{/ /}$piece of the action.

\section{The Topological Effective Theory.}

The restriction to the vacuum subspace of $S_{\perp}$ eliminates the local gravitational degrees of freedom: only the global variations of the remaining longitudinal modes $X^{a}$ can be physically relevant. In this section we will construct the topological field theory that describes their dynamics.

From the above discussion we learn that, to leading order in $g_{\perp}$, we can restrict ourselves to the special class of vacuum field configurations such that

$$
S_{\perp}[h, g]=0
$$

This leads to the equations

$$
\begin{aligned}
\partial_{\alpha} h_{i j} & =0 \\
R_{g} & =0,
\end{aligned}
$$

which single out the vacuum solutions in which no graviton waves propagate. These solutions are of the general form

$$
\begin{aligned}
h_{i j} & =h_{i j}(y) \\
g_{\alpha \beta} & =\eta_{a b} \partial_{\alpha} X^{a} \partial_{\beta} X^{b}
\end{aligned}
$$


The variables $X^{a}$ are maps of the two-dimensional $x$-plane onto itself and vary in the transversal $y$ directions. Here the transversal metric $h_{i j}(y)$ is allowed to be arbitrary and will be treated as a fixed classical background.

Along with the metric, the ghost fields are also constrained to solve the equation of motion of the weakly coupled $S_{\perp}$ piece of the ghost action (2.8). This equation for the anti-ghost, $\nabla^{\alpha} b_{i \alpha}=0$, is solved by

$$
b_{i \alpha}=\epsilon_{\alpha \beta} \partial^{\beta} b_{i}
$$

where the new anti-ghosts $b_{i}$ are unrestricted field variables. Substituting the parametrizations (3.3) and (3.4) into the Einstein action gives the effective action for the new variables $X^{a}, c^{\alpha}$ and $b^{i}$.

To write this reduced action, it is convenient to introduce the vector fields $V_{i}^{\alpha}$ via the relation

$$
\partial_{i} X^{a}+V_{i}^{\alpha} \partial_{\alpha} X^{a}=0
$$

These variables $V_{i}^{\alpha}$ describe the flow of the $X^{a}$ fields in the $y$-direction, and are analogous to the fluid velocity in fluid mechanics. The transversal derivatives of the metric can be expressed in terms of $V_{i}^{\alpha}$ as

$$
\partial_{i} g^{\alpha \beta}=\nabla^{\alpha} V_{i}^{\beta}+\nabla^{\beta} V_{i}^{\alpha}
$$

Here and in the following we keep the $X^{a}$ dependence implicit in $g_{\alpha \beta}$ and $V_{i}^{\alpha}$. After substituting this into (2.5) and using the identity $\epsilon_{\alpha \delta} \epsilon_{\beta \gamma}=\epsilon_{\alpha \gamma} \epsilon_{\beta \delta}-\epsilon_{\alpha \beta} \epsilon_{\gamma \delta}$ we find that the action naturally splits in two contributions

$$
\begin{gathered}
S=S_{1}+S_{2} \\
S_{1}=\int \sqrt{h} \sqrt{g}\left(R_{h}+\epsilon_{\alpha \gamma} \epsilon_{\beta \delta} \nabla^{\alpha} V_{i}^{\beta} \nabla^{\gamma} V^{i \delta}\right) \\
S_{2}=\int \sqrt{h} \sqrt{g}\left(-\frac{1}{2}\left(\epsilon_{\alpha \beta} \partial^{\alpha} V_{i}^{\beta}\right)^{2}+b^{i} \epsilon_{\alpha \beta} \partial^{\alpha} \partial_{i} c^{\beta}\right) .
\end{gathered}
$$

Notice that, when we insert (3.3) and (3.5), this action is highly non-linear in the fundamental fields $X^{a}$. It describes, as promised, a topological field theory: there are no local physical degrees of freedom. The first term is invariant under local variations of the $X^{a}$-fields, and can in fact be written as a total derivative. The second 
term in (3.7), when regarded as a two-dimensional field theory in the transversal $y$-plane, represents a kind of two-dimensional topological sigma model [⿴囗十). In this interpretation the target space of the model is given by the space of diffeomorphisms of the $x^{\alpha}$ plane.

Both terms in the action (3.7) are invariant under the nilpotent BRST symmetry

$$
\begin{aligned}
\delta X^{a} & =\varepsilon c^{\beta} \partial_{\beta} X^{a}, \\
\delta b^{i} & =\varepsilon\left(\epsilon_{\alpha \beta} \partial^{\alpha} V^{i \beta}+c^{\beta} \partial_{\beta} b^{i}\right), \\
\delta c^{\alpha} & =\varepsilon c^{\beta} \partial_{\beta} c^{\alpha},
\end{aligned}
$$

under which $V_{i}^{\alpha}$ transforms as

$$
\delta V_{i}^{\alpha}=\varepsilon\left(-\partial_{i} c^{\alpha}+c^{\beta} \partial_{\beta} V_{i}^{\alpha}-V_{i}^{\beta} \partial_{\beta} c^{\alpha}\right)
$$

This nilpotent symmetry removes all local fluctuations of the $X^{a}$-field, and is a remainder of the BRST-invariance of the original theory, as can be seen as follows.

The original anti-ghost field $b_{i \alpha}$ transforms under the BRST-symmetry into the $R_{i \alpha}$ components of the Ricci-tensor, which are the constraints associated with our gauge choice. Therefore, the redefinition (3.4) of the ghost field is compatible with BRST-invariance only if $R_{i \alpha}$ is also of the form

$$
R_{i \alpha}=\epsilon_{\alpha \beta} \partial^{\beta} R_{i}
$$

Using that the background satisfies the field equations of $S_{\perp}$, which gives that $\nabla^{\alpha} R_{i \alpha}=-\nabla^{j} G_{i j}=0$, one deduces that (3.10) indeed holds. Explicitly, one has

$$
R_{i}=\epsilon_{\alpha \beta} \partial^{\alpha} V_{i}^{\beta}
$$

The BRST-symmetry of the reduced action (3.7) thus directly follows from that of the gauge fixed Einstein action. It effectively imposes the constraint $R_{i}=0$, which tells us that the flows $V_{i}$ are curl free.

Let us end this subsection with one final comment. The attentive reader may have worried a little about the fact that the changes of variables from $g_{\alpha \beta}$ to $X^{a}$ and from $b_{i \alpha}$ to $b^{i}$, both require some Jacobians to correctly treat the functional measure. 
It turns out, however, that these two Jacobians cancel each other. Explicitly, if we define the measures of $g_{\alpha \beta}$ and $X^{a}$ through

$$
\begin{aligned}
\left\|\delta g_{\alpha \beta}\right\|^{2} & =\int \sqrt{h} \sqrt{g} \epsilon^{\alpha \gamma} \epsilon^{\beta \delta} \delta g_{\alpha \beta} \delta g_{\gamma \delta}, \\
\left\|\delta X^{a}\right\|^{2} & =\int \sqrt{h} \sqrt{g} \eta_{a b} \delta X^{a} \delta X^{b}
\end{aligned}
$$

one obtains from (2.10) that the measures of $g_{\alpha \beta}$ and $X^{a}$ are related via

$$
\left[d g_{\alpha \beta}\right]=\left[d X^{a}\right]\left|\operatorname{det}\left(\epsilon_{\alpha \beta} \nabla^{\beta}\right)\right|^{2}
$$

Similarly, since the ghosts are anti-commuting, we deduce from (3.4) that

$$
\left[d b_{i \alpha}\right]=\left[d b_{i}\right]\left|\operatorname{det}\left(\epsilon_{\alpha \beta} \nabla^{\beta}\right)\right|^{-2}
$$

So the total Jacobian of the substitution $\left(g_{\alpha \beta}, b_{i \alpha}\right) \rightarrow\left(X^{a}, b^{i}\right)$ is indeed equal to 1 .

\section{The Action for the Boundary Values}

Let us now ask ourselves what physical degrees of freedom are described by the action (3.7). For definiteness, we will from now on consider the situation where the $x^{\alpha}$-coordinates parametrize (a part of) the two-dimensional Minkowski plane $M$. Thus our space has a boundary $\partial M$ in this direction, consisting of four asymptotic regions, two belonging to the past null infinity $\mathcal{I}^{-}$and two constituting the future null infinity $\mathcal{I}^{+}$(see fig. 1). Now we notice that local variations of the fields $X^{a}$ are not physical, since they correspond to diffeomorphisms and are cancelled by the BRST-symmetry. Therefore the only possible physical degrees of freedom are the boundary values

$$
\bar{X}^{a} \equiv X_{\mid \partial M}^{a}
$$

We will allow these boundary values $\bar{X}^{a}$ to be arbitrary, because, as we will discuss below, this enables us to incorporate matter into the theory. Furthermore, to make them into true physical observables, we impose the usual restriction that gauge 


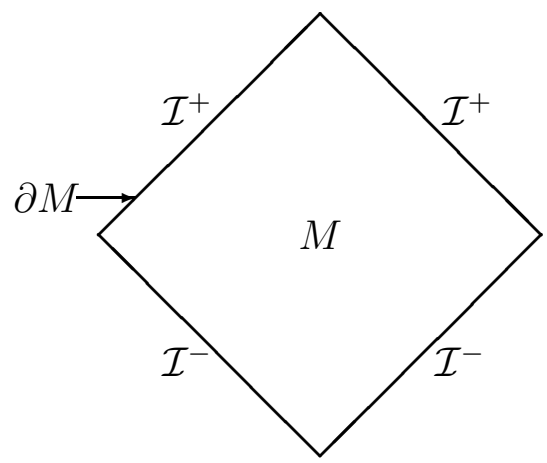

Fig. 1: The boundary $\partial M$ of the two-dimensional parameter space $M$ of the longitudinal coordinates $x^{\alpha}$ has four components corresponding to the four asymptotic regions of the Minkowski plane.

transformations of the theory, i.e. diffeomorphisms, asymptotically go to the identity transformation. After gauge fixing this translates into the asymptotic condition that the $c$-ghost vanishes on $\partial M$, and thus the $\bar{X}^{a}$ are BRST-invariant.

We will now show that for physical field configurations the action (3.7) can be expressed purely in terms of $\bar{X}^{a}$. This is most evident for the first term $S_{1}[X]$ : it is easy to see that it is the integral of a total derivative. Hence

$$
S_{1}[X]=S_{\partial M}[\bar{X}]
$$

One finds

$$
S_{\partial M}[\bar{X}]=\oint_{\partial M} d x^{\alpha} \int \sqrt{h} \epsilon_{a b}\left(R_{h} \bar{X}^{a} \partial_{\alpha} \bar{X}^{b}+\partial_{i} \bar{X}^{a} \partial_{\alpha} \partial^{i} \bar{X}^{b}\right) .
$$

The second term of the action (3.7) plays the role of the gauge fixing term to the action, needed to eliminate the redundancy caused by the topological nature of the first term. Following a standard argument, often applied in topological field theory 四, we can use the symmetry (3.8) of the action to restrict the functional integral to the BRST-invariant field configurations. This implies that we may put the ghost fields $c^{\alpha}$ equal to zero, and in addition impose the constraint

$$
\epsilon_{\alpha \beta} \partial^{\alpha} V_{i}^{\beta}=\epsilon^{\alpha \beta} \partial_{\alpha}\left(\partial_{i} X^{a} \partial_{\beta} X_{a}\right)=0 .
$$

When we insert this into (3.7) we find that the $S_{2}$-piece of the action vanishes 
identically. We conclude, therefore, that for physical field configurations the total action indeed reduces to the simple boundary term (4.3).

Finally, a more precise specification of what we mean by the asymptotic values $\bar{X}^{a}$ of $X^{a}$ is perhaps in order here, since a priori at least one of them becomes infinite when we approach the null infinities $\mathcal{I}^{ \pm}$. The point here is that, as we will see, only variations of the asymptotic values of the $X^{a}$ are physically relevant. Hence it is sufficient for our purposes to consider, instead of the full Minkowski plane, only a finite region $M$, large enough so that variations $\delta X^{a}$ at the boundary $\partial M$ are equal to the asymptotic variations. This procedure will be implicitly adopted in the following sections.

\subsection{Coupling to Matter}

We will now discuss the coupling of external matter to the gravitational field, and show that it can also be represented entirely in terms of the boundary values $\bar{X}^{a}$. We consider the situation in which asymptotically all particle momenta are in the $x^{\alpha}$-direction, so that at the boundary $\partial M$ all components except $T_{\alpha \beta}$ vanish.

First let us assume that also in the interior region $T_{\alpha \beta}$ are the only non-vanishing components. Its conservation law then reads

$$
\nabla^{\alpha} T_{\alpha \beta}=0
$$

This tells us that the matter source term $S_{m}[g]$ is invariant under diffeomorphisms of $x^{\alpha}$. In terms of the $X^{a}$ field variables this implies that $S_{m}[g]$ is invariant under local variations, and indeed only depends on the boundary values $\bar{X}^{a}$. To write this dependence, it is convenient to represent the incoming and outgoing matter at the boundary $\partial M$ by means of a momentum flux $P_{a \alpha}$, defined in terms of the stress-energy tensor via

$$
T_{\alpha \beta}=P_{a \alpha} \partial_{\beta} X^{a}
$$

Then the variation of the matter contribution with respect to $X^{a}$ is

$$
\begin{aligned}
\delta S_{m}[g(X)] & =2 \int \sqrt{h} \sqrt{g} \nabla^{\alpha}\left(P_{a \alpha} \delta X^{a}\right) \\
& =2 \oint_{\partial M} d x^{\alpha} \int \sqrt{h} \epsilon_{\alpha}{ }^{\beta} P_{a \beta} \delta \bar{X}^{a} .
\end{aligned}
$$


Thus, the matter momentum in- and out-flux couples directly to the variations of the boundary values $\bar{X}^{a}$.

In general, however, it is possible that in the interior of $M$ also the mixed components $T_{i \alpha}$ of the stress tensor become non-vanishing. Although in this case we have to refine the above derivation of the matter coupling, it in fact turns out that the final result (4.7) remains unaltered. Let us indicate how this goes. The conservation equation (4.5) is now modified to

$$
\nabla^{\alpha} T_{\alpha \beta}+\nabla^{i} T_{i \beta}=0
$$

Because we assume $T_{i j}=0$, we know that $T_{i \alpha}$ in addition satisfies $\nabla^{\alpha} T_{i \alpha}=0$. It is therefore of the form

$$
T_{i \alpha}=\epsilon_{\alpha \beta} \partial^{\beta} J_{i}
$$

Here $J_{i}$ can be identified with the current associated with the boosts $\delta X^{a}=\epsilon_{b}^{a} X^{b}$ in the $X$-plane. Namely, by integrating (4.8) over the plane $M$ against $\xi^{\beta}=\partial^{\beta} X^{a} X^{b} \epsilon_{a b}$ and inserting the redefinitions (4.6) and (4.9) we deduce that for all $y$

$$
\nabla_{i} \int_{M} \sqrt{g} J^{i}=\oint_{\partial M} d x^{\alpha} \epsilon_{\alpha}^{\beta} P_{\beta}^{a} \bar{X}^{b} \epsilon_{a b}
$$

which expresses the conservation of angular momentum.

When $J_{i}$ is non-vanishing, the matter action $S_{m}[g]$ is no longer invariant under local variations of $X^{a}$. However, coordinate invariance tells us that this local $X^{a}$ dependence must cancel when we include the gravitational interaction. To see this, consider the BRST-variation of the matter action $S_{m}[g]$. It can be expressed in terms of the current $J_{i}$ as follows

$$
\begin{aligned}
\delta_{b r s t} S_{m}[g] & =2 \varepsilon \int \sqrt{h} \sqrt{g} T_{\alpha \beta} \nabla^{\alpha} c^{\beta} \\
& =2 \varepsilon \int \sqrt{h} \sqrt{g} J^{i} \epsilon_{\alpha \beta} \partial^{\alpha} \partial_{i} c^{\beta},
\end{aligned}
$$

where we used (4.8) and (4.9). Thus the combined matter and gravitational action is BRST-invariant, provided we include $J^{i}$ in the transformation law (3.8) of the anti-ghost. As a consequence the constraint (4.4) on physical field configurations is replaced by

$$
\epsilon_{\alpha \beta} \partial^{\alpha} V_{i}^{\beta}=J_{i}
$$


With this modification we can now follow the argument of the preceding subsection to show that, under the above physical constraint, the dependence of $S_{m}[g]$ on local variations of $X^{a}$ indeed cancels against that of the second term of the gravitational action (3.7). Hence also in this case the entire coupling to matter is described by equation (4.7).

\section{The Scattering Matrix}

In this section we will determine the amplitudes describing the gravitational scattering of the matter particles. First we like to summarize the result of the above analysis. We have shown that, due to the BRST-invariance, the functional integral over the fields $X^{a}, b^{i}$, and $c^{\alpha}$ is concentrated on the space of physical field configurations, satisfying the constraint (4.12). For given boundary values $\bar{X}^{a}$, this constraint uniquely fixes the interior values of $X^{a}$, and thus all dynamics is reduced to the boundary $\partial M$. It will be convenient to introduce a 'time'-variable $\tau$ which parametrizes the coordinates $x^{\alpha}(\tau)$ on $\partial M$. Notice that, since $\partial M$ is closed, $\tau$ is periodic. The action that describes the dynamics of the boundary values and its coupling to matter is

$$
S_{\partial M}[X]=S[X]+2 \int d \tau \int \sqrt{h} P_{a, \tau} X^{a}
$$

with

$$
S[X]=\int d \tau \int \sqrt{h} \epsilon_{a b} \frac{\partial X^{a}}{\partial \tau}\left(\Delta_{h}-R_{h}\right) X^{b},
$$

where $\Delta_{h}$ denotes the scalar Laplacian in the transversal $y$-plane. Here we used (4.7) to represent the matter coupling in terms of the momentum density $P_{a, \tau}=$ $\epsilon_{\beta}^{\alpha} P_{a \alpha} \partial_{\tau} x^{\beta}$. In (5.1) and from now on we drop the bar on the boundary values of $X^{a}$. In the rest of this section it is understood that all the fields are defined on $\partial M$.

\subsection{QuANTUM MEChanics of the BOUndary VALUES.}

The boundary theory (5.1) can naturally be regarded as a (non-relativistic) $2+1$ dimensional field theory. Indeed to obtain the scattering amplitudes we will still 
need to perform the remaining functional integral over the $X^{a}$, and it is therefore a natural step to consider the quantum mechanics of this boundary theory. For this it is a fortunate fact that the action (5.2) is quadratic in the fields $X^{a}$, especially since it was derived from the highly non-linear Einstein action. Canonical quantization is therefore straightforward. It leads to the following commutation relations

$$
\left[X^{a}\left(y_{1}\right), X^{b}\left(y_{2}\right)\right]=i \epsilon^{a b} f\left(y_{1}, y_{2}\right)
$$

where

$$
\left(\Delta_{h}-R_{h}\right) f\left(y_{1}, y_{2}\right)=\delta^{(2)}\left(y_{1}, y_{2}\right)
$$

defines the Green function of the modified Laplacian $\left(\Delta_{h}-R_{h}\right)$. This commutator previously appeared in the work of 't Hooft [2]. Intuitively it can be understood from the Einstein equation (5.9), which relates the coordinate fields $X^{a}$ to their canonical conjugate momenta. Let us denote by $\mathcal{H}$ the Hilbert space in which the commutator algebra (5.3) is realized; vectors in $\mathcal{H}$ can be represented as functionals of $X^{+}(y)$ or of $X^{-}(y)$. Note that, since the action is invariant under reparametrizations in $\tau$, there is no Hamiltonian, i.e. $H=0$.

The matter coupling is described in terms of 'vertex operators' of the form

$$
V(P)=\operatorname{Pexp}\left(2 i \int d \tau \int \sqrt{h} P_{a, \tau}(\tau, y) X^{a}(y)\right)
$$

Here the path-ordering is necessary, as the $X^{a}(y)$ do not mutually commute. The momentum flux $P_{a, \tau}$ will for the moment still be treated as a classical quantity, but in the next subsection, when we study the scattering matrix, $P_{a, \tau}$ becomes a quantum mechanical operator in the Hilbert space of the matter theory. The idea is that in this way, by first computing the expectation value of the general vertex operator (5.5), we obtain a generating functional for all scattering amplitudes. Since the boundary time $\tau$ is periodic, this expectation value is given by the trace over the Hilbert space $\mathcal{H}$

$$
\langle V(P)\rangle=\operatorname{tr}_{\mathcal{H}}\left(\operatorname{Pexp}\left[2 i \oint d \tau \int \sqrt{h} P_{a, \tau} X^{a}\right]\right) .
$$

The result can be computed from the commutation relations (5.3) via a simple application of the $\mathrm{CBH}$-formula, or alternatively, by using the relation with the 
functional integral. One finds

$$
\langle V(P)\rangle=\exp \left[i \int_{\tau<\tau^{\prime}} d \tau d \tau^{\prime} \int \sqrt{h} \epsilon^{a b} P_{a, \tau}\left(\Delta_{h}-R_{h}\right)^{-1} P_{b, \tau^{\prime}}\right]
$$

In addition we obtain a delta-function imposing the condition that the total momentum flux at the transversal position $y$ vanishes

$$
\oint d \tau P_{a, \tau}(\tau, y)=0
$$

This condition, which is due to the invariance of $S[X]$ under $\tau$-independent shifts of $X^{a}$, guarantees that the result (5.7) does not depend on the base-point of the $\tau$-integration.

The result (5.7) for the generating function is exactly given by the semi-classical expression, $\exp \left(i S\left[X_{c l}\right]\right)$, where $X_{c l}$ solves the classical equation of motion

$$
\left(\Delta_{h}-R_{h}\right) \frac{\partial X^{a}}{\partial \tau}=\epsilon^{a b} P_{b, \tau}
$$

This equation, which is in fact the remainder of the Einstein equation, only involves the values of the coordinate fields $X^{a}$ and momenta $P_{a}$ on the boundary $\partial M$. Thus with our formalism we have succeeded in computing the Einstein action of the relevant classical solution, without needing to integrate the equation of motion for the interior region.

In principle the classical configuration for $X^{a}$ in the interior region is determined by the physical constraint (4.12) involving the current $J_{i}$. The integral of (4.12) over the two-dimensional parameter space $M$ gives for all $y$ the relation

$$
\oint_{\partial M} d \tau \frac{\partial X^{a}}{\partial \tau} \partial_{i} X_{a}(y)=\int_{M} \sqrt{g} J_{i}(y)
$$

where we used that $\epsilon_{\alpha \beta} \partial^{\alpha} V_{i}^{\beta}=\epsilon^{\alpha \beta} \partial_{\alpha}\left(\partial_{i} X^{a} \partial_{\beta} X_{a}\right)$. As a consistency check one can verify, using the equation of motion (5.9), that this relation implies the conservation law 4.10 ). 


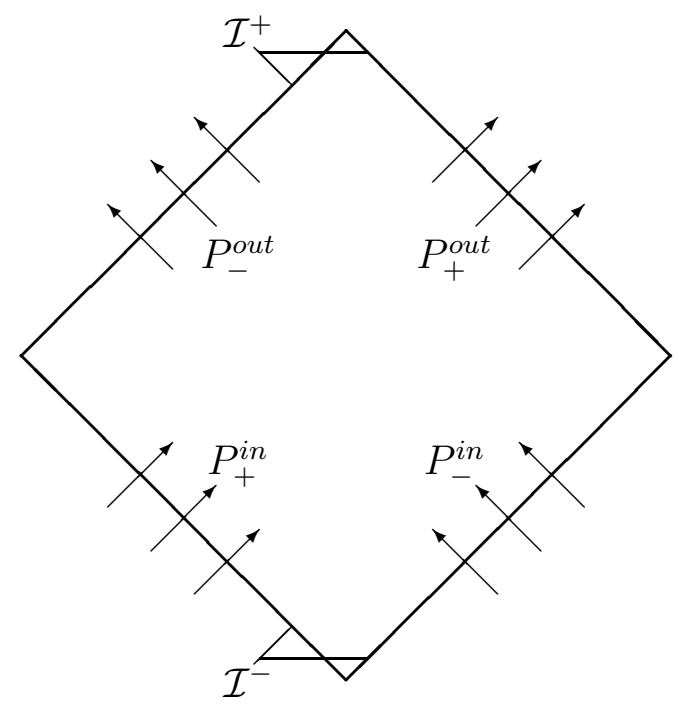

Fig. 2: The amplitude (5.13) is the $S$-matrix element between the state with momentum flux $P_{a}^{i n}$ at the in-region $\mathcal{I}^{-}$and the state of given out-going flux $P_{a}^{\text {out }}$ at $\mathcal{I}^{+}$.

\subsection{Description of the Scattering Matrix}

Let us now determine the scattering matrix for (point-like) matter particles. Since we assume their masses to be negligible compared to the Planck mass, they are divided into left-movers with momenta $p_{-}^{i}$ and right-movers with momenta $p_{+}^{j}$. Now, it is important to note that the left- and right-movers come in from or go out to different asymptotic regions of space-time, so that the boundary $\partial M$ is divided into four different components, on each of which either $P_{-, \tau}$ or $P_{+, \tau}$ vanishes, (see fig. 2). Correspondingly, the vertex operator (5.5) representing the momentum flux decomposes into the product of four vertex operators, where, for example, the operator representing the right-moving incoming particles has the form

$$
V\left(P_{+}^{i n}\right)=\exp \left(2 i \int \sqrt{h} P_{+}^{i n}(y) X^{+}(y)\right),
$$

where

$$
P_{+}^{i n}(y)=\int_{\mathcal{I}^{-}} d \tau P_{+, \tau}(\tau, y)
$$

is the total incoming momentum at the transversal position $y$. The scattering amplitude is obtained by specializing the general formula (5.7) to the present situation. 
This gives the result

$$
\left\langle V\left(P_{+}^{\text {in }}\right) V\left(P_{-}^{\text {in }}\right) V^{*}\left(P_{+}^{\text {out }}\right) V^{*}\left(P_{-}^{\text {out }}\right)\right\rangle=S(P) \delta\left(P_{a}^{\text {out }}-P_{a}^{\text {in }}\right),
$$

where

$$
S(P)=\exp \left(i \int \sqrt{h} \int \sqrt{h} \eta^{a b} P_{a}\left(y_{1}\right) f\left(y_{1}, y_{2}\right) P_{b}\left(y_{2}\right)\right)
$$

is the scattering matrix.

The variables $P_{a}(y)$ act as quantum mechanical operators in the matter Hilbert space, and can be used to characterize the in- and out states. Because the operators $P_{a}(y)$ mutually commute, we can find a basis of eigenstates in the in- and outHilbert space. Such a basis is obtained by specifying for each particle its longitudinal momentum $p_{a}^{(i)}$ and transversal coordinate $y^{(i)}$. On the $N$-particle state $\left|p^{(i)}, y^{(i)}\right\rangle$, the momentum operator $P_{a}(y)$ has the eigenvalue

$$
P_{a}(y)\left|p_{a}^{(i)}, y^{(i)}\right\rangle=\sum_{i=1}^{N} p_{a}^{(i)} \delta\left(y, y^{(i)}\right)\left|p^{(i)}, y^{(i)}\right\rangle .
$$

Since the $S$-matrix (5.14) is expressed purely in terms of the $P_{a}(y)$, it is also diagonal in the same basis, with eigenvalues

$$
S\left(p^{(i)}, y^{(i)}\right)=\prod_{i \neq j} \exp \left(i \eta^{a b} p_{a}^{(i)} p_{b}^{(j)} f\left(y^{(i)}, y^{(j)}\right)\right) .
$$

Equations (5.14) and (5.16) give the final result for the scattering matrix. Notice that the $N$-particle amplitude factorizes into the product of two-particle amplitudes, which tells us that there are no three- or higher point interactions. In the next section we will discuss the two-particle process in more detail.

We observe that the $S$-matrix element (5.16) can be written in a form similar to a string amplitude as follows

$$
S\left(p^{(i)}, y^{(i)}\right)=\int[d X] e^{i S_{s t r}[X]} \prod_{i} e^{2 i p_{a}^{(i)} X^{a}\left(y^{(i)}\right)},
$$

where $S_{\text {str }}$ denotes the two-dimensional action for a string embedded in a $1+1$ dimensional space-time (cf. [2])

$$
S_{s t r}[X]=\int d^{2} y \sqrt{h}\left(h^{i j} \partial_{i} X^{a} \partial_{j} X_{a}+R_{h} X^{a} X_{a}\right) .
$$


An unusual feature is the factor $i$ in front of the action, even though the "world sheet', the $y$-plane, has Euclidean signature.

Another difference with the usual string amplitudes is that there is no integration over the positions of the vertex operators, nor over the metric $h_{i j}$. The reason for not integrating over these variables is that we assume that for large $x^{\alpha}$ we are in the classical regime, where general covariance is broken in the $y$-direction via some vacuum expectation value $\left\langle h_{i j}\right\rangle=h_{i j}$. One can imagine, however, some physical situations where general covariance remains unbroken. In this case we do have to integrate over $h_{i j}$ and the formal analogy with string theory becomes almost exact.

\section{The Two-Particle Amplitude}

In this section we will take a more detailed look at the two-particle scattering process, and discuss its geometrical interpretation. In the following we will assume that the transversal metric is flat $h_{i j}=\delta_{i j}$. In this case the Green function $f\left(y_{1}, y_{2}\right)$ becomes

$$
f\left(y_{1}, y_{2}\right)=-\log \left|y_{1}-y_{2}\right|^{2}
$$

\subsection{The Shock Wave Geometry}

The scattering matrix obtained in the previous subsection has a concrete physical interpretation in terms of gravitational shock waves [1, 5]. To exhibit this interpretation, we first observe that the N-particle amplitude factorizes into the product of two-particle amplitudes, given by

$$
S_{12}=\exp \left(2 i p_{1}^{-} p_{2}^{+} f\left(y_{1}, y_{2}\right)\right)
$$

where $p_{i}^{ \pm}$and $y_{i}$ are the momentum and transversal coordinate of particle $i$. This two-particle result is obtained from (5.14) by choosing the in-flux of momentum at $\mathcal{I}^{-}$of the following form

$$
\begin{aligned}
& P_{i n}^{-}(x, y)=p_{1}^{-} \delta\left(x^{+}-x_{1}^{+}\right) \delta\left(y-y_{1}\right) \\
& P_{i n}^{+}(x, y)=p_{2}^{+} \delta\left(x^{-}-x_{2}^{-}\right) \delta\left(y-y_{2}\right)
\end{aligned}
$$




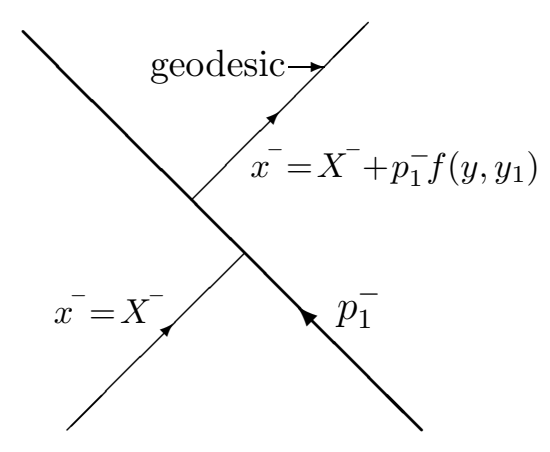

Fig. 3: The shock wave geometry of a fast left-moving particle with momentum $p_{1}^{-}$and transversal position $y_{1}$ has for all $y$ a discontinuity at the $x^{\alpha}$-trajectory of the particle.

The corresponding classical solution of (5.9) is given by

$$
\begin{aligned}
& X_{i n}^{-}=x^{-}-p_{1}^{-} \theta\left(x^{+}-x_{1}^{+}\right) f\left(y, y_{1}\right), \\
& X_{i n}^{+}=x^{+}-p_{2}^{+} \theta\left(x^{-}-x_{2}^{-}\right) f\left(y, y_{2}\right) .
\end{aligned}
$$

These classical field configurations for $X_{i n}^{+}$and $X_{i n}^{-}$both exhibit a discontinuity at the $x^{\alpha}$ trajectory of one of the particles. These discontinuities are the well-known gravitational impulsive wave, or 'shock wave', solutions to the Einstein equation obtained in [5]. The form of this shock wave geometry for a single particle is indicated in fig. 3. It shows that a right-moving geodesic is shifted by an amount $p_{1}^{-} f\left(y, y_{1}\right)$ when it passes through the $x^{\alpha}$-trajectory of the fast left-moving particle with momentum $p_{1}$.

The formula (6.4) only describes the two gravitational shock waves in the inregion, i.e. before they collided. It is clear that in the out-region the solution will become more complicated. To solve for the complete metric describing the two colliding shock waves indeed appears to be a rather non-trivial problem. The technical difficulty here is that after the two particle trajectories cross each other, the position of each shock wave is shifted by the discontinuity of the other. This distorts the waves in such a way that they are no longer stable solutions of the Einstein equation. What we know, however, is that the out-flux $P_{\tau, a}(\tau, y)$ at $\mathcal{I}^{+}$must, by momentum conservation, yield the same integrated momentum distribution $P_{a}(y)$ as the in-flux. This is all information we need to compute the scattering amplitude 
$S_{12}$ via the general formula (5.14).

Finally, let us note that the amplitude (6.2) has been derived in an alternative way in [1] by considering the effect of the shock wave of one of the two colliding particles on the wave packet of the other. This reasoning makes use of the fact that one can go to a frame where one of the particles moves slowly, so that only the gravitational field of the other fast moving particle needs to be considered. As an operator in the Hilbert space of the slow particle, the $S$-matrix (6.2) can then be recognized as the generator of the coordinate shift caused by the shock wave of the fast particle.

\subsection{Partial Wave Analysis}

The two-particle process is special because in this case our geometrical setup in fact does not break Lorentz invariance: the division into longitudinal and transversal directions puts no restrictions on the momenta of the two particles, but is defined in terms of them. We will now indeed show that the two-particle $S$-matrix and differential cross section can be given in a Lorentz invariant form. Our approach is based on a partial wave analysis of the scattering amplitude, and can be regarded as a refinement of the method of [1]. It was found in [1] that the two-particle amplitude possesses an infinite number of poles for imaginary $P^{2}$, which were conjectured to indicate the presence of bound states. One of our aims is to determine whether these poles already occur at the level of partial waves or not.

To write the $S$-matrix (6.2) in manifestly Lorentz invariant form, we can first replace $p_{1}^{+} p_{2}^{-}$by $p_{1}^{\mu} p_{2 \mu}$. Next, let us introduce the total linear and angular momentum operators

$$
\begin{aligned}
P^{\mu} & =p_{1}^{\mu}+p_{2}^{\mu}, \\
J_{\mu} & =\frac{1}{|P|} \epsilon_{\mu \nu \lambda \sigma} p_{1}^{\nu} p_{2}^{\lambda}\left(x_{1}^{\sigma}-x_{2}^{\sigma}\right),
\end{aligned}
$$

and express $p_{1}^{\mu} p_{2 \mu}$ and the impact parameter $\left|y_{1}-y_{2}\right|$ in terms of these quantities as (here we use that $p_{1}^{2}=p_{2}^{2}=0$ )

$$
\begin{gathered}
2 p_{1}^{\mu} p_{2 \mu}=P^{2}, \\
\left|y_{1}-y_{2}\right|^{2}=J^{2} / P^{2} .
\end{gathered}
$$


Notice that this implies that as a quantum mechanical operator the impact parameter has a discrete spectrum.

The two-particle scattering operator is conveniently expressed in terms of the mutually commuting operators $P$ and $J$ as follows

$$
S_{12}=\left(\frac{J^{2}}{P^{2}}\right)^{-i P^{2}}
$$

Hence the S-matrix is diagonal in the partial waves basis of eigenmodes $|P, l, m\rangle$ of $P, J^{2}$ and $J_{3}$, and can be represented as

$$
S_{12}|P, l, m\rangle=e^{2 i \delta_{l}(P)}|P, l, m\rangle
$$

where the phase shift $\delta_{l}(P)$ is given by

$$
\delta_{l}(P)=-\frac{1}{2} P^{2} \log \left(\frac{l(l+1)}{P^{2}}\right)
$$

This result gives the exact leading behaviour of the scattering matrix for large angular momentum $l$, that is

$$
l(l+1) \gg P^{2}
$$

with $P^{2}$ measured in Planck units. Notice therefore that if $P^{2}$ is small our approximation is reliable for all partial waves, except for the $s$-wave. Indeed, the above result for the phase shift becomes singular for $l=0$. Notice further that, for fixed $l$, the $S$-matrix does not exhibit any poles in $P^{2}$.

From the knowledge of the phase shifts $\delta_{l}(P)$ one can compute the differential cross-section via

$$
\begin{aligned}
\frac{d \sigma}{d \theta}(\theta) & =\left|f_{P}(\theta)\right|^{2} \\
f_{P}(\theta) & =\frac{1}{2 i|P|} \sum_{l=0}^{\infty}(2 l+1)\left(e^{2 i \delta_{l}(P)}-1\right) P_{l}(\cos \theta) .
\end{aligned}
$$

Inserting the result (6.10) (and restricting the sum in (6.12) to $l>0$ ) we obtain an expression for the amplitude $f_{P}(\theta)$ which is reliable for small scattering angles $\theta$. 
To extract this leading behaviour we make use of the following asymptotic formula, which becomes exact for large $l$ and small $\theta$

$$
P_{l}(\cos \theta) \rightarrow J_{0}\left((2 l+1) \sin \frac{1}{2} \theta\right)
$$

where $J_{0}$ is the first Bessel function. Let us now re-introduce the impact parameter $y=\sqrt{l(l+1)} /|P|$ and approximate the sum over $l$ by the integral over $y$. The resulting integral can be performed and gives in this approximation the following leading behaviour

$$
\begin{aligned}
f_{P}(\theta) & =-i|P| \int_{0}^{\infty} d y y^{1-2 i P^{2}} J_{0}\left(2 y|P| \sin \frac{1}{2} \theta\right) \\
& =\frac{|P|}{2 \sin ^{2} \frac{1}{2} \theta} \frac{\Gamma\left(1-i P^{2}\right)}{\Gamma\left(1+i P^{2}\right)}\left(P^{2} \sin ^{2} \frac{1}{2} \theta\right)^{i P^{2}} .
\end{aligned}
$$

This formula agrees with the result obtained in [1].

The most striking feature of the scattering amplitude (6.14) is the occurrence of poles at the center of mass energies $P^{2}=-i N$, in Planck units. However, it is clear from the above derivation that they do not correspond to any resonant states of quantum gravity, but are produced by the replacement of the sum over $l$ by the integral over $y$. If there would exist resonances with definite spin, the corresponding poles would already have been visible in the phase shifts $\delta_{l}(P)$. The poles in (6.14) come from the $y \rightarrow 0$ part of the integral and disappear when this part of the integral is cut-off and replaced by a sum. Hence, assuming that the $s$-wave contribution has no poles, the original expression (6.12) for the scattering amplitude is analytic for all values of $P^{2}$. We conclude therefore that result (6.14) describes the correct small $\theta$ behaviour of the amplitude as long as $\operatorname{Im} P^{2}>-1$; beyond this point subleading terms in (6.12) become important.

\section{Concluding Remarks}

In this paper we have described a systematic method for studying forward scattering in quantum gravity. It exploits the presence of the small dimensionless parameter $g_{\perp}=\left(l_{p l} / l_{\perp}\right)^{2}$, in terms of which one can do perturbation theory. The final 
result (5.14) for the scattering amplitude is valid only to leading order in $g_{\perp}$, but is non-perturbative in the other dimensionless coupling constant $g_{/ /}=\left(l_{p l} / l_{/ /}\right)^{2}$. It can therefore be applied for arbitrarily large center of mass energies.

We have shown that the relevant gravitational modes that mediate between the high energy particles are described by means of a topological field theory and that their dynamics and the coupling to matter is formulated in terms of the boundary values of $X^{a}$. This result is universal in the sense that it only depends on general covariance and dimensional analysis, and also applies in the presence of a cosmological constant or curvature squared terms. Curvature squared terms will, however, modify the boundary action (4.3) by higher derivative terms. The fundamental property that remains is the absence of local dynamics at short distances. This is a reflection of the fact that in the $x^{\alpha}$-direction general covariance in unbroken, and in this sense we have truly dealt with the 'topological phase' of quantum gravity.

The fact that the leading order amplitude (5.14) is unitary, tells us that to this order no gravitational radiation is produced in the scattering process. Indeed, the coupling to gravitons is of lower order in the momentum transfer. It is possible to compute these higher order corrections due to the presence of gravitons, although at some point one is bound to encounter the problem of non-renormalizability of quantum gravity. String models of quantum gravity are likely to avoid this problem, but we expect that these string modifications will not drastically alter the leading order result (see, however, [6]).

One of the more intriguing conclusions of our paper is the commutation relation (5.3) between the coordinate fields $X^{a}$. Its geometrical origin lies in the fact that the discontinuities describing the gravitational shock waves (6.4) of the left- and rightmoving particles are in a way incompatible: the shift caused by the left-mover causes an uncertainty in the location of the right-mover, and vice versa, at the point where the two $x^{\alpha}$ trajectories intersect. One may speculate that the commutator hints towards the existence of a new quantum gravitational uncertainty principle, which could have radical consequences. Indeed, 't Hooft has proposed that, by taking this uncertainty principle serious, one can construct an $S$-matrix for the successive black hole formation and evaporation process [2].

In view of this application, it would be very interesting to generalize our analysis to the case where $x$, instead of being a cartesian coordinate, represents a radial coordinate $r$. This corresponds to the physical situation in which the momenta of all particles are directed to the same point $(r=0)$ in space. However, here we 
will meet a new difficulty, since for very small values of $r$ our assumption about the transversal momenta being small becomes unattainable. Therefore additional modes of the gravitational field need to be included in the analysis. One indeed expects that in this situation black hole formation may take place, and then e.g. the mass of the black hole becomes a dynamical variable.

\section{Appendix: The Einstein Action in the Gauge (2.2)}

In this Appendix we outline the derivation of the formula (2.5)-(2.6) for the Einstein action in the gauge

$$
g_{\mu \nu}=\left(\begin{array}{cc}
g_{\alpha \beta} & 0 \\
0 & h_{i j}
\end{array}\right)
$$

Here and in the following $\mu, \nu$ run from 0 to $3, \alpha, \beta$ from 0 to 1 and $i, j$ from 2 to 3 . Under this decomposition the Ricci scalar of $g_{\mu \nu}$ reads

$$
R^{(4)}=g^{\alpha \beta} g^{\gamma \delta} R_{\alpha \gamma \beta \delta}+h^{i j} h^{k l} R_{i k j l}+2 g^{\alpha \beta} h^{i j} R_{\alpha i \beta j}
$$

where $R_{\mu \nu \lambda \sigma}$ is the Riemann tensor

$$
R_{\mu \nu \lambda \rho}=\partial_{\lambda} \Gamma_{\mu \rho \nu}-\partial_{\rho} \Gamma_{\mu \lambda \nu}+\Gamma_{\mu \lambda \sigma} \Gamma_{\rho \nu}^{\sigma}-\Gamma_{\mu \rho \sigma} \Gamma_{\lambda \nu}^{\sigma}
$$

Inserting the explicit form of the following Christoffel symbols

$$
\begin{gathered}
\Gamma_{i \alpha \beta}=-\Gamma_{\alpha \beta i}=-\frac{1}{2} \partial_{i} g_{\alpha \beta} \\
\Gamma_{\alpha i j}=-\Gamma_{i j \alpha}=-\frac{1}{2} \partial_{\alpha} h_{i j}
\end{gathered}
$$

while keeping $\Gamma_{\alpha \beta}^{\gamma}$ and $\Gamma_{i j}^{k}$ implicit, we find for the first two terms in (A.2)

$$
\begin{aligned}
g^{\alpha \beta} g^{\gamma \delta} R_{\alpha \gamma \beta \delta} & =R_{g}-\frac{1}{4} h^{i j} \partial_{i} g_{\alpha \beta} \partial_{j} g_{\gamma \delta}\left(g^{\alpha \delta} g^{\beta \gamma}-g^{\alpha \beta} g^{\gamma \delta}\right) \\
h^{i j} h^{k l} R_{i k j l} & =R_{h}-\frac{1}{4} g^{\alpha \beta} \partial_{\alpha} h_{i j} \partial_{\beta} h_{k l}\left(h^{i l} h^{j k}-h^{i j} h^{k l}\right)
\end{aligned}
$$


where $R_{g}$ and $R_{h}$ are the scalar curvatures of $g$ and $h$ considered as two-dimensional metrics. The last term in (A.2) becomes

$$
\begin{aligned}
2 g^{\alpha \beta} h^{i j} R_{\alpha i \beta j}= & \frac{1}{2} h^{i j} \partial_{i} g_{\alpha \beta} \partial_{j} g_{\gamma \delta}\left(g^{\alpha \delta} g^{\beta \gamma}-g^{\alpha \beta} g^{\gamma \delta}\right) \\
& +\frac{1}{2} g^{\alpha \beta} \partial_{\alpha} h_{i j} \partial_{\beta} h_{k l}\left(h^{i l} h^{j k}-h^{i j} h^{k l}\right) \\
& +\frac{1}{\sqrt{g}} \nabla^{i} \partial_{i} \sqrt{g}+\frac{1}{\sqrt{h}} \nabla^{\alpha} \partial_{\alpha} \sqrt{h}
\end{aligned}
$$

Here the last two terms contribute total derivatives to the Einstein action, and can therefore be omitted. Adding the remaining terms and using

$$
\begin{aligned}
g^{\alpha \delta} g^{\beta \gamma}-g^{\alpha \beta} g^{\gamma \delta} & =\epsilon^{\alpha \gamma} \epsilon^{\beta \delta} \\
h^{i l} h^{j k}-h^{i j} h^{k l} & =\epsilon^{i k} \epsilon^{j l}
\end{aligned}
$$

leads to the result (2.5)-(2.6) for the Einstein action.

\section{References}

[1] G. 't Hooft, Phys. Lett. B198 (1987) 61; Nucl. Phys. B304 (1988) 867.

[2] G. 't Hooft, Nucl. Phys. B335 (1990) 138.

[3] E. Witten, Commun. Math. Phys. 117 (1988) 353

[4] E. Witten, Commun. Math. Phys. 118 (1988) 411.

[5] P. Aichelburg and R. Sexl, J. Gen. Rel. Grav. 2 (1971) 303;

T. Dray and G. 't Hooft, Nucl. Phys. B 253 (1985) 173.

[6] D. Amati, M. Ciafaloni and G. Veneziano, Int. J. of Mod. Phys. 3 (1988) 1615 